\title{
Indication for initiation of mechanical circulatory support impacts survival of infants with shunted single-ventricle circulation supported with extracorporeal membrane oxygenation
}

\author{
Catherine K. Allan, MD, ${ }^{a}$ Ravi R. Thiagarajan, MBBS, MPH, ${ }^{a}$ Pedro J. del Nido, MD, ${ }^{b}$ Stephen J. Roth, MD, MPH, \\ Melvin C. Almodovar, MD, and Peter C. Laussen, $\mathrm{MBBS}^{\mathrm{a}}$
}

Objectives: The use of extracorporeal membrane oxygenation to support patients with shunted single-ventricle physiology has been controversial. Variable survivals are reported in a number of small case series. We sought to evaluate outcomes and identify predictors of survival for patients with shunted single-ventricle physiology who require extracorporeal membrane oxygenation support.

Methods: We retrospectively reviewed the medical records of all patients aged less than 1 year with shunted single-ventricle physiology who were supported with extracorporeal membrane oxygenation at Children's Hospital Boston between 1996 and 2005. Survivors and nonsurvivors were compared with respect to demographics, diagnosis, operative variables, indication for extracorporeal membrane oxygenation, and extracorporeal membrane oxygenation variables.

Results: Forty-four infants with shunted single-ventricle physiology were supported with extracorporeal membrane oxygenation. Diagnoses included hypoplastic left heart syndrome (24), other single-ventricle lesions (12), and pulmonary atresia/ intact ventricular septum or a variant (8). Overall survival to discharge was $48 \%$. Indication for extracorporeal membrane oxygenation was the strongest predictor of survival to discharge, with $81 \%$ of patients cannulated for hypoxemia but only $29 \%$ of those cannulated for hypotension surviving to discharge. Specifically, patients cannulated for shunt obstruction had the highest survival (83\%).

Conclusions: Overall survival to discharge for patients with shunted single-ventricle physiology is similar to survival reported in the Extracorporeal Life Support

From the Departments of Cardiology and Cardiac Surgery, ${ }^{\text {b }}$ Children's Hospital Boston and Harvard Medical School, Boston, Mass; and Division of Pediatric Cardiology, Lucile Packard Children's Hospital and Stanford University School of Medicine, Palo Alto, Calif.

Received for publication Aug 24, 2006; revisions received Oct 26, 2006; accepted for publication Nov 1, 2006.

Address for reprints: Catherine K. Allan, MD, Children's Hospital Boston, Department of Cardiology, 300 Longwood Avenue, Boston, MA 02115 (E-mail: catherine. allan@cardio.chboston.org).

J Thorac Cardiovasc Surg 2007;133:660-7

$0022-5223 / \$ 32.00$

Copyright () 2007 by The American Association for Thoracic Surgery

doi:10.1016/j.jtcvs.2006.11.013 Organization registry for all infants supported with cardiac extracorporeal membrane oxygenation. Thus, shunted single-ventricle physiology should not be considered a contraindication to extracorporeal membrane oxygenation. Patients cannulated for hypoxemia, particularly shunt thrombosis, had markedly improved survival compared with those supported primarily for hypotension/cardiovascular collapse. Survival did not differ depending on anatomic diagnosis.

$\mathrm{V}$ enoarterial extracorporeal membrane oxygenation (ECMO) is commonly used for circulatory support in pediatric cardiac surgical patients with refractory low cardiac output, persistent hypoxemia, arrhythmias, cardiac arrest, or failure to wean from cardiopulmonary bypass (CPB). ${ }^{1-4}$ Survival to hospital discharge for pediatric patients receiving cardiac ECMO support reported to the Extracorporeal Life Support Organization (ELSO) registry for 2004 was 41\%. ${ }^{5}$ However, the ELSO registry report and reports of ECMO outcomes from individual institutions have shown considerable variability in survival because of the differences in anatomic diagnosis, surgical procedure, and ECMO indication. The vari- 


$$
\begin{aligned}
& \text { Abbreviations and Acronyms } \\
& \begin{aligned}
\mathrm{BT} & =\text { Blalock-Taussig } \\
\mathrm{CICU} & =\text { cardiac intensive care unit } \\
\mathrm{CPB} & =\text { cardiopulmonary bypass } \\
\mathrm{CPR} & =\text { cardiopulmonary resuscitation } \\
\mathrm{ECMO} & =\text { extracorporeal membrane oxygenation } \\
\mathrm{ELSO} & =\text { Extracorporeal Life Support Organization } \\
\mathrm{HLHS} & =\text { hypoplastic left heart syndrome } \\
\mathrm{IVS} & =\text { intact ventricular septum } \\
\mathrm{PA} & =\text { pulmonary atresia } \\
\text { RVDCC } & =\text { right ventricle-dependent coronary } \\
& \text { circulation }
\end{aligned}
\end{aligned}
$$

ability in outcomes may reflect differences in patient selection, timing of ECMO cannulation, and ECMO management. Early reports of the use of cardiac ECMO suggested that outcomes for patients with single-ventricle circulation are substantially worse than those with 2-ventricle circulation. ${ }^{6,7}$ On the basis of these reports, some centers have considered single-ventricle circulation to be a relative contraindication to use of ECMO. ${ }^{7,8}$ However, more recent studies have reported survival as high as $64 \%$ in this group of patients. ${ }^{9}$

The use of ECMO for the support of patients with aortopulmonary shunts with or without systemic outflow tract reconstruction (functionally univentricular circulation with an aortopulmonary shunt, which for simplicity will be termed "shunted single-ventricle circulation" throughout the text), such as after the Norwood procedure, poses a number of unique problems beyond those experienced with ECMO support in patients with 2-ventricle circulation. There is an ongoing need to balance systemic and pulmonary perfusion on ECMO to prevent myocardial and systemic ischemia caused by excessive runoff into the low-resistance pulmonary bed through the shunt. In addition, the volume load placed on the single ventricle may impair the recovery of myocardial function after CPB. Some or all of these factors may contribute to the increased morbidity and mortality previously reported for ECMO support in these patients.

The available literature regarding outcomes and risk factors for mortality in this patient population is limited by small patient numbers and lack of systematic review of clinical variables. Here we report the results of our experience with the use of ECMO for support of patients with single-ventricle physiology with aortopulmonary shunts. We sought to determine factors associated with survival to hospital discharge in this high-risk group of patients supported with ECMO to identify subsets of patients most likely to benefit from ECMO support.

\section{Materials and Methods \\ Patients}

We conducted a retrospective review of the ECMO database at Children's Hospital Boston to identify all infants ( $<1$ year of age) with an aortopulmonary shunt with or without systemic or pulmonary outflow tract reconstruction who were supported with ECMO between 1996 and 2005. Patients who had undergone the Norwood procedure with a right ventricle to pulmonary artery conduit rather than a modified Blalock-Taussig (BT) shunt were excluded from this study because we thought they represent a physiologically different group. We retrospectively reviewed medical records, daily ECMO records, and cardiac catheterization and operative reports to collect data on demographics, diagnoses, surgeries, indications for ECMO, details of ECMO course, and outcomes. Approval for medical record review was obtained from the Children's Hospital Boston Committee on Clinical Investigation. The need for informed consent was waived.

\section{Extracorporeal Membrane Oxygenation Management}

Direct intrathoracic cannulation was carried out for patients placed on ECMO in the operating room after CPB and for those placed on ECMO in the cardiac intensive care unit (CICU) during the immediate postoperative period. Patients who had not previously undergone median sternotomy or who were temporally remote from surgery were placed on ECMO support through the internal jugular vein and common carotid artery. For patients placed on ECMO in the operating room or electively in the CICU, ECMO was initiated using a blood-primed circuit. For patients requiring urgent ECMO cannulation, such as during cardiopulmonary resuscitation (CPR), a crystalloid-primed circuit, available in the CICU at all times, was used. Patients cannulated during CPR had ice packs placed around the head during resuscitation, and mild systemic hypothermia $\left(34.5^{\circ} \mathrm{C}-35.5^{\circ} \mathrm{C}\right)$ was maintained during the first 24 hours of ECMO support. After stabilization on ECMO, diagnostic studies were undertaken to ascertain the reason for needing ECMO support. Diagnostic modalities, including echocardiography, cardiac catheterization, and mediastinal/shunt exploration, were used as clinically indicated. The decision to investigate and choice of diagnostic modality, daily supervision of medical care, and decisions regarding further therapeutic or surgical interventions were at the discretion of the cardiac intensivist and surgeon. Management of the ECMO circuit, including anticoagulation and replacement of blood products, was conducted according to institutional practice guidelines for the care of patients on ECMO.

The initial target for ECMO flow for all patients was 100 to 200 $\mathrm{mL} / \mathrm{kg} / \mathrm{min}$. Most patients were maintained on full flow for at least 24 hours after cannulation before attempts were made to wean flows. Investigations for residual anatomic lesions were conducted before attempts to wean from ECMO support. For patients placed on ECMO using a crystalloid-primed circuit, ultrafiltration was used to remove crystalloid volume, which was replaced with packed red blood cells to increase the hematocrit to $35 \%$. Continued use of ultrafiltration for management of intravascular volume or to aid free water clearance was at the discretion of the cardiac intensivist.

Whenever possible, it was our practice to maintain shunt patency during ECMO support to reduce the risk of pulmonary 
ischemia-reperfusion injury and to avoid shunt thrombosis. However, in patients exhibiting decreased systemic perfusion or with delayed clearance of lactate, despite increasing ECMO flows and adjusting sweep gases to augment systemic oxygen delivery, 1 or more surgical clips was placed to decrease the diameter of the shunt and thus limit pulmonary blood flow.

\section{Data Analysis}

Patients were categorized into 2 groups according to primary indication for ECMO: (1) those who underwent cannulation for circulatory collapse (hypotension, failure to wean from $\mathrm{CPB}$, or cardiac arrest after profound hypotension) and (2) those who underwent cannulation for hypoxemia. For patients whose preECMO period was characterized by both hypoxemia and hypotension, 2 authors (C. K. A. and R. R. T.) reviewed the physicians' notes and intensive care unit charts and categorized patients into 1 of the 2 ECMO indication groups on the basis of consensus opinion on which was the primary indication. Patients who were successfully decannulated and survived to discharge were compared with those who died with respect to demographic variables, ECMO indication, preoperative and operative variables, details of the ECMO course, and complications. For patients who had 2 or more periods on ECMO, only data from the first ECMO run were included in the analysis.

Data were analyzed using the Statistical Package for the Social Sciences (SPSS v 12.0, Inc, Chicago, Ill). Continuous variables were compared using the Mann-Whitney test. Categoric variables were compared using the chi-square test, and the Fisher exact test was used when the individual expected count in 1 or more categories was less than 5. In a multivariate logistic regression model, we evaluated the association of ECMO indication and survival to hospital discharge after adjusting for all other variables that were significantly associated with survival.

\section{Results}

\section{Patient Demographics and Diagnoses}

Forty-four infants (15\% of 283 cardiac ECMO uses during the study period) with shunted single-ventricle circulation underwent 47 runs of ECMO support. Patient demographics are summarized in Table 1. Hypoplastic left heart syndrome (HLHS) $(\mathrm{n}=24 ; 55 \%)$ was the most frequent diagnosis. Twelve patients $(27 \%)$ had non-HLHS single-ventricle anatomy, and 8 patients (18\%) had pulmonary atresia (PA) or a variant. All patients received ECMO support only postoperatively, except for 1 patient with HLHS and a restrictive atrial septum who was placed on ECMO preoperatively after left atrial perforation during attempted atrial septostomy in the cardiac catheterization laboratory. This patient also required ECMO support after a Norwood operation for failure to wean from CPB. Because of the short duration of preoperative support, only the postoperative support details were included in this analysis. Surgical procedures performed included the Norwood operation in 29 patients $(66 \%)$ and BT shunt placement without systemic outflow reconstruction in 15 patients $(34 \%)$, representing $10 \%$ of all Norwood procedures $(\mathrm{n}=288)$ and $8 \%$ of all BT
TABLE 1. Patient demographics

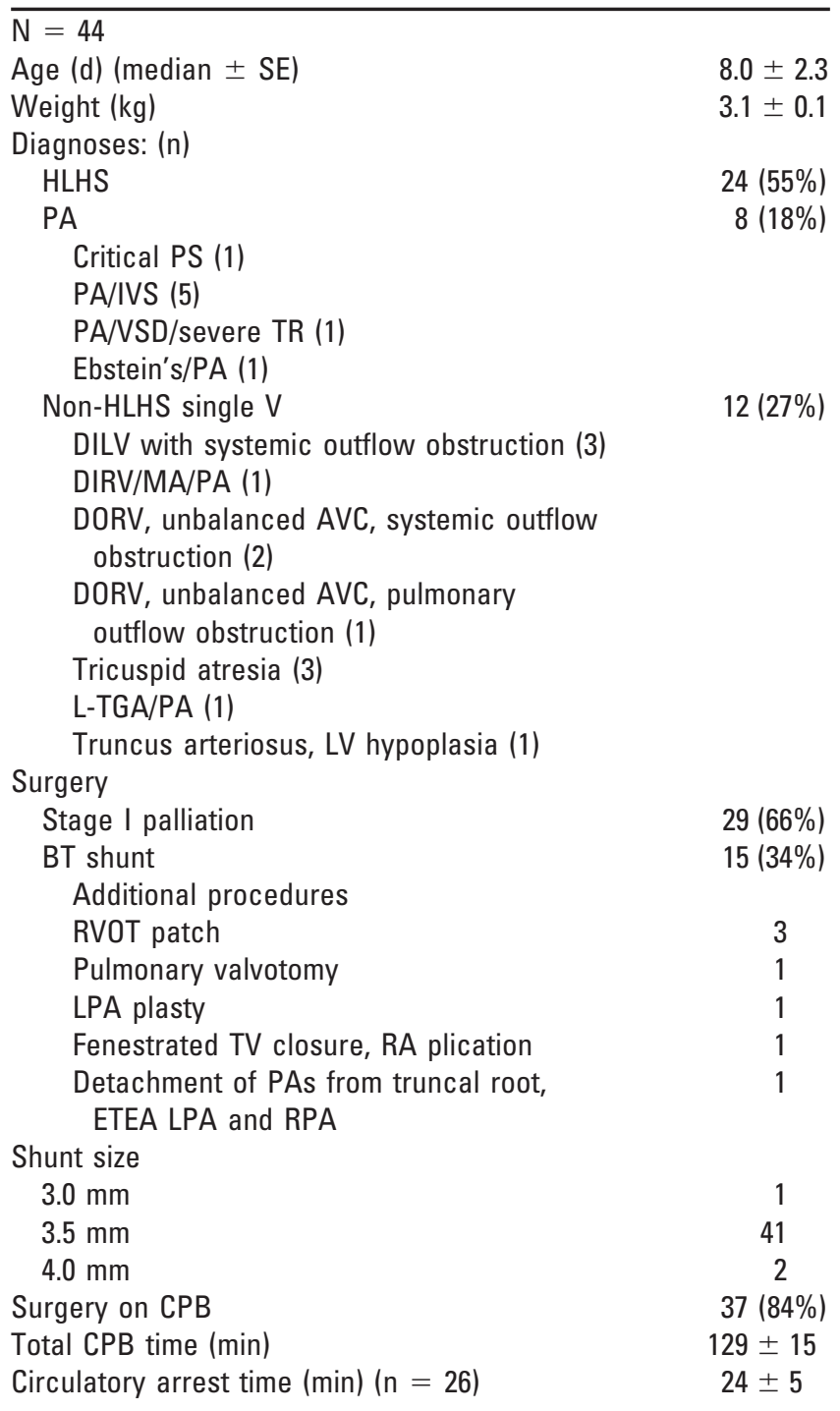

$S E$, Standard error; HLHS, hypoplastic left heart syndrome; $P S$, pulmonary stenosis; PA/IVS, pulmonary atresia/intact ventricular septum; $V S D$, ventricular septal defect; $T R$, tricuspid regurgitation; DILV, double inlet left ventricle; DIRV, double inlet right ventricle; $M A$, mitral atresia; DORV, double outlet right ventricle; AVC, atrioventricular canal; L-TGA, I-transposition of the great arteries; $L V$, left ventricle; $B T$, Blalock-Taussig; $L P A$, left pulmonary artery; $T V$, tricuspid valve; $R A$, right atrium; $P A s$, pulmonary arteries; $E T E A$, end-to-end anastomosis; $R P A$, right pulmonary artery; $C P B$, cardiopulmonary bypass; RVOT, right ventricular outflow tract.

shunts $(\mathrm{n}=179)$ performed in our institution during that time period. Of the 15 patients who underwent BT shunt placement without systemic outflow reconstruction, 9 patients underwent surgery with CPB (2 with significant hypoxemia or hemodynamic instability at the time of operation and 7 requiring additional surgical intervention) (Table 1). One patient who underwent a Norwood operation for double outlet right ventricle, complete atrioventricular canal, and coarctation of the aorta also underwent reimplantation of an 


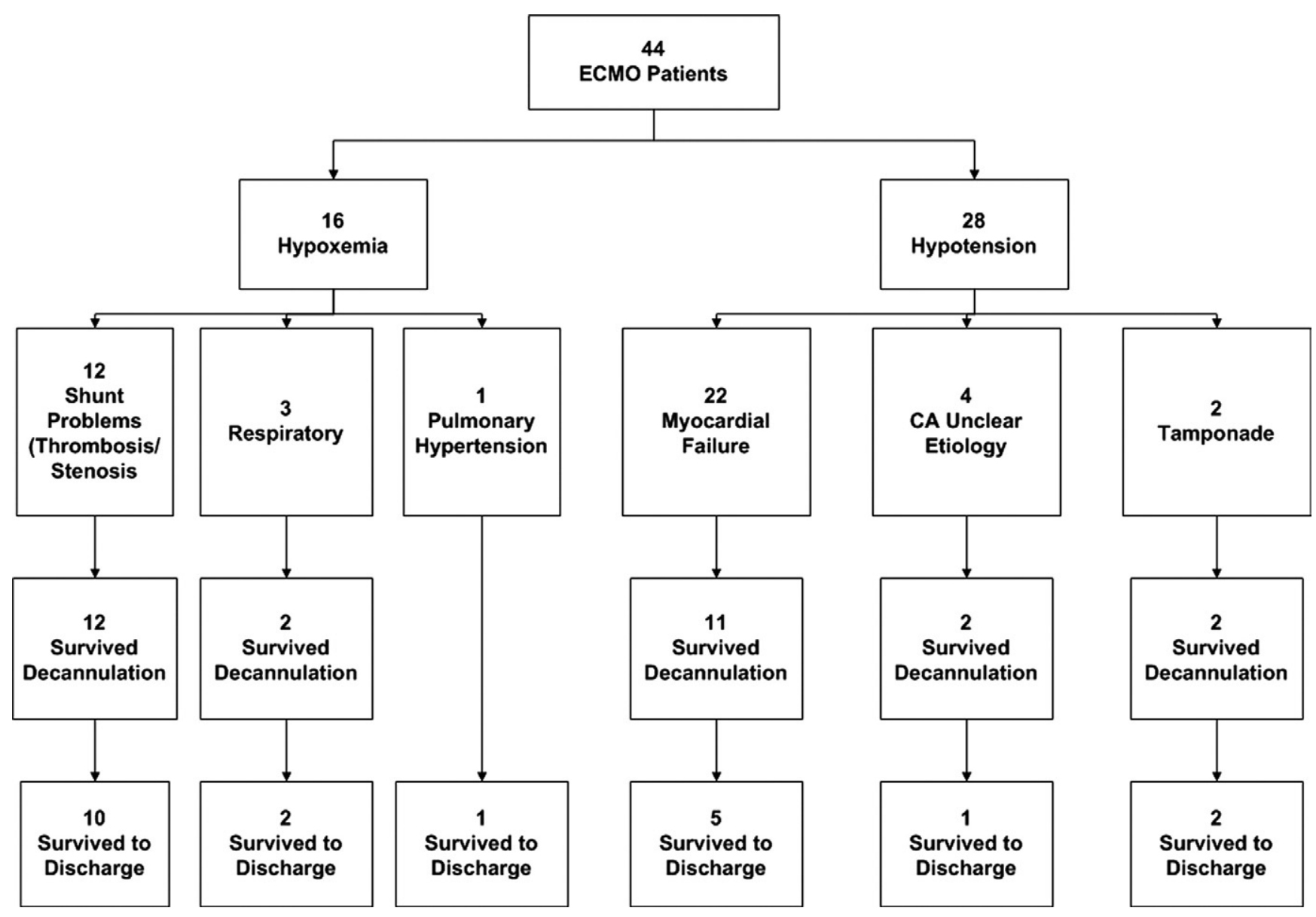

Figure 1. Indication for cannulation and outcomes for 44 patients with shunted single-ventricle physiology who were supported with ECMO. ECMO, Extracorporeal membrane oxygenation; CA, cardiac arrest.

anomalous left coronary artery arising from the pulmonary artery.

\section{Indications for Extracorporeal Membrane \\ Oxygenation}

Patient characteristics and outcomes based on ECMO indication are shown in Table 1 and Figure 1. Twenty-eight patients $(64 \%)$ received ECMO support for circulatory collapse. Of these, 5 patients $(11 \%)$ had failed to wean from CPB secondary to hypotension despite escalating inotropic support. Twenty-one patients were placed on ECMO support for refractory low cardiac output (17) or sudden cardiac arrest (4) in the CICU. ECMO support was initiated after circulatory collapse secondary to tamponade in the remaining 2 patients.

Sixteen patients $(36 \%)$ received ECMO support for profound hypoxemia, 12 of whom had documented shunt abnormalities on subsequent investigations. One patient had hypoxemia secondary to preexistent pulmonary vascular disease that was unresponsive to conventional therapies including inhaled nitric oxide, and 3 patients had hypoxemia secondary to parenchymal lung disease. Thirty-one patients $(70 \%)$ were receiving CPR during the initiation of ECMO support. ECMO support was initiated in the CICU in the majority of patients $(\mathrm{n}=37 ; 84 \%)$.

Details of Extracorporeal Membrane Oxygenation Course and Patient Evaluation on Extracorporeal Membrane Oxygenation

The median duration of ECMO support was 125 hours (range 8-530 hours). The details of ECMO support for survivors and nonsurvivors are shown in Table 2.

\section{Evaluation of Patients on Extracorporeal}

Membrane Oxygenation

Echocardiography was the primary modality used to evaluate function and anatomy for patients on ECMO. In addition, all 10 patients cannulated for shunt thrombosis under- 
TABLE 2. Demographic, surgical, intraoperative, and extracorporeal membrane oxygenation variables for survivors versus nonsurvivors

\begin{tabular}{lccc}
\hline & $\begin{array}{c}\text { Survivors (21) } \\
\text { (median } \pm \text { SE) }\end{array}$ & $\begin{array}{c}\text { Nonsurvivors (23) } \\
\text { (median } \pm \text { SE) }\end{array}$ & $\boldsymbol{P}$ \\
\hline Age (d) & $11.5 \pm 2.1$ & $6 \pm 4.0$ & .55 \\
Weight (kg) & $3.2 \pm 0.1$ & $2.9 \pm 0.1$ & .17 \\
Diagnosis & & & .09 \\
$\quad$ HLHS & 13 & 11 & \\
PA & 1 & 7 & \\
Single ventricle, & 7 & 5 & \\
$\quad$ non-HLHS & & & .46 \\
Surgery & & & \\
Stage I & 15 & 14 & \\
BT shunt & 6 & 9 & \\
Shunt size/weight & $1.09 \pm 0.05$ & $1.21 \pm 0.05$ & .30 \\
$\quad$ (mm/kg) & & & \\
CPB time (min) & $129 \pm 15$ & $108 \pm 22$ & .38 \\
CA time (min) (n $=26)$ & $25 \pm 5$ & $15 \pm 7$ & .65 \\
Peak OR lactate & $7.5 \pm 0.9$ & $8.5 \pm 1.3$ & .40 \\
Failed to wean from & $2(11 \%)$ & $3(15 \%)$ & 1.00 \\
$\quad$ CPB & & & \\
Indication for ECMO & & & .001 \\
$\quad$ Hypoxemia & 13 & 3 & \\
$\quad$ Hypotension & 8 & 20 & \\
CPR & $13(62 \%)$ & $18(78 \%)$ & .33 \\
Hours on ECMO & $114 \pm 15$ & $160 \pm 23$ & .001 \\
Shunt clipped & $4(20 \%)$ & $14(61 \%)$ & .007 \\
Peak ECMO lactate & $11.6 \pm 1.9$ & $13.5 \pm 1.4$ & .18 \\
Lowest pH & $7.18 \pm 0.03$ & $7.22 \pm 0.04$ & .38 \\
Lowest P0 & $32 \pm 3$ & $35 \pm 3$ & .01 \\
BUN & $32 \pm 3$ & & \\
Creatinine & $24 \pm 3$ & $45 \pm 4$ & $<.001$ \\
AST & $0.7 \pm 0.1$ & $1.2 \pm 0.1$ & $<.001$ \\
ALT & $105 \pm 61$ & $159 \pm 108$ & .001 \\
SET & $22 \pm 20$ & $24 \pm 18$ & .27 \\
\hline
\end{tabular}

$S E$, Standard error; HLHS, hypoplastic left heart syndrome; $P A$, pulmonary atresia; $B T$, Blalock-Taussig; $C P B$, cardiopulmonary bypass; $C A$, circulatory arrest; $O R$, operating room; $C P R$, cardiopulmonary resuscitation; $E C M 0$, extracorporeal membrane oxygenator; $B U N$, blood urea nitrogen; $A S T$, aspartate aminotransferase; $A L T$, alanine aminotransferase.

went surgical shunt exploration at the time of ECMO cannulation, with shunt thrombus directly visualized in 9 of 10 patients. Thrombus was not seen in 1 patient, but suctioning of the distal end of the shunt at the time of shunt exploration led to a dramatic improvement in the pulmonary perfusion, supporting the diagnosis of shunt thrombosis.

Fourteen patients underwent catheterization while on ECMO to investigate the cause of the event leading to ECMO or reason for inability to wean from ECMO. Catheterization diagnosed shunt narrowing in 1 patient and right subclavian artery stenosis in 2 patients, all of whom had hypoxemia as an ECMO indication. Each of these patients underwent operative intervention based on catheterization findings, and all 3 successfully weaned from ECMO and survived to discharge. Catheterization was diagnostic in an additional 4 patients. Two with HLHS had left ventriclecoronary fistulae and resulting myocardial ischemia. One patient with severe Ebstein's anomaly and PA who had previously undergone BT shunt placement and right ventricular outflow tract patch had confirmation of a retrograde circular shunt. This patient underwent main pulmonary artery ligation but still failed to wean from ECMO. The fourth patient with PA/intact ventricular septum (IVS) and right ventricle-dependent coronary circulation (RVDCC) had a large right ventricular thrombus impairing flow across the tricuspid valve and therefore coronary perfusion.

\section{Shunt Management}

Surgical clips were placed on the BT shunt to limit pulmonary blood flow in 17 of 44 patients (39\%). Lactate clearance was delayed ( $>48$ hours) for more patients who required shunt clipping than for those who had a patent shunt throughout ECMO (71\% vs 39\%, respectively). This difference approached statistical significance $(P=.06)$. Fewer survivors than nonsurvivors had shunt clips placed (19\% vs $57 \%, P=.011)$. As expected, more patients cannulated for cardiovascular collapse versus hypoxemia required shunt clipping (54\% vs $6 \%, P=.024)$. Two patients cannulated for hypoxemia required shunt clipping. One was a premature infant with hypoxemia secondary to lung disease of prematurity who had excessive runoff through a $3.5-\mathrm{mm}$ BT shunt. The second was a $2.1-\mathrm{kg}$ infant who required placement of 3 clips on a $3.5-\mathrm{mm}$ BT shunt to separate from $\mathrm{CPB}$. Shunt thrombosis subsequently developed in this infant, and after shunt thrombectomy, a single clip was placed on the shunt to facilitate weaning from ECMO.

\section{Outcomes}

Thirty patients $(68 \%)$ were successfully weaned from ECMO, and 21 patients (48\%) survived to hospital discharge. Table 2 summarizes the preoperative, operative, and ECMO variables for survivors versus nonsurvivors. Age, weight, and type of surgery did not differ significantly between survivors and nonsurvivors, nor did BT shunt size normalized to patient weight (BT shunt size [millimeters]/ weight [kilograms]). For patients who underwent BT shunt placement without systemic outflow reconstruction, survival did not differ between those who underwent shunt placement on $\mathrm{CPB}$ or had additional surgical intervention at the same time (eg, right ventricular outflow tract patch) versus those who did not. ECMO indication was significantly different between survivors and nonsurvivors, with $81 \%$ of patients cannulated for hypoxemia and only $29 \%$ of those cannulated for circulatory collapse surviving to discharge $(P=.001)$. Pre-ECMO minimum $\mathrm{PAO}_{2}$ was lower for patients who survived to discharge (32 vs $36 \mathrm{~mm} \mathrm{Hg}$, $P=.01)$, consistent with the fact that patients cannulated 
for hypoxemia had improved survival compared with those cannulated for circulatory collapse. BT shunt size normalized to patient weight did not differ between those cannulated for hypoxemia versus those cannulated for circulatory collapse (data not shown). The duration of ECMO support was shorter (56 \pm 15 hours vs $160 \pm 23$ hours, $P=.001)$ for survivors. The longest duration of ECMO support for a survivor not requiring transplantation was 303 hours compared with 530 hours for nonsurvivors. The lowest $\mathrm{pH}$ around the time of ECMO cannulation and maximum lactate on ECMO did not differ between survivors and nonsurvivors. Indices of end-organ dysfunction on ECMO, including peak blood urea nitrogen, creatinine, and aspartate aminotransferase, were significantly lower for survivors (Table 2), although neither of these groups showed clinically significant elevation of these laboratory parameters. In a multivariate logistic regression model, after adjustment for variables significantly associated with survival in univariate analysis (blood urea nitrogen, creatinine, and aspartate aminotransferase), hypoxemia compared with circulatory collapse as an indication for ECMO remained significantly associated with survival to discharge (odds ratio for survival 10.8 [95\% CI 1.2-99.9, $P=.04]$ ).

Although diagnosis did not differ significantly between survivors and nonsurvivors, there was a trend toward higher mortality in patients with PA variants $(P=.09)$. Survival was $14 \%$ for patients with PA variants compared with 54\% for those with HLHS and 58\% for those with non-HLHS single-ventricle anatomy.

Among the 30 patients successfully weaned from ECMO, 3 who were initially cannulated for low cardiac output syndrome went on to require a second period of ECMO support during the same hospitalization. Indications for second ECMO course included hypoxemia (shunt thrombosis) (1), low cardiac output syndrome (1), and progressive renal and respiratory insufficiency (1). Two of these patients were successfully decannulated, and 1 patient survived to discharge.

\section{Neurologic Complications of Extracorporeal Membrane Oxygenation}

Head ultrasounds were obtained on all infants as part of the routine ECMO management protocol. Ten patients had evidence of hemorrhage or infarct on head ultrasound. Nine of these patients did not survive to discharge, although management decisions were made on the basis of head ultrasound findings in only 1 of these patients. This infant with parenchymal and intraventricular hemorrhage had ECMO discontinued because of head ultrasound findings and died shortly thereafter despite maximal medical management.

\section{Bidirectional Glenn and Fontan Completion}

Follow-up data are available for patients who returned to our institution for further care. Twelve of 21 survivors have gone on to Fontan completion; 4 patients have undergone the bidirectional Glenn procedure, 3 of whom are awaiting Fontan completion and 1 for whom further follow-up data are not available. One patient underwent transplantation during the initial hospitalization and is alive today. One patient underwent conversion to a 2-ventricle circulation and is alive at follow-up. Three patients died before the bidirectional Glenn procedure; 2 died suddenly at home, and 1 died in hospice care with severe pulmonary hypertension.

\section{Discussion}

In our study of 44 patients with shunted single-ventricle circulation supported with ECMO, we show a $48 \%$ survival to hospital discharge. We also show that indication for ECMO is significantly associated with survival to discharge, with $81 \%$ of patients supported with ECMO for an indication of hypoxemia compared with $29 \%$ of those supported for cardiovascular collapse surviving to hospital discharge.

The use of ECMO to support patients with shunted single-ventricle circulation has been controversial. Some centers have successfully used prophylactic mechanical support without an oxygenator after the Norwood operation to allow a period of myocardial recovery after CPB. ${ }^{10}$ However, for patients with shunted single-ventricle circulation who need urgent or semi-urgent ECMO support in the postoperative period, the literature provides conflicting data as to whether mortality for these patients is comparable to mortality for all patients supported with cardiac ECMO. Our survival to discharge of $48 \%$ is comparable to $41 \%$ cumulative survival for all neonatal and pediatric cardiac ECMO cases reported to the ELSO registry and to the cumulative survival to discharge of $53 \%$ for all patients supported with cardiac ECMO in our institution (Peter Laussen, MBBS, personal communication, 2005).

Cumulative survival for infants with HLHS who were supported with ECMO reported to the ELSO registry between 1996 and 2000 is $28 \%$ and has not changed significantly over this time period. ${ }^{11}$ Survival at individual centers has recently been reported to be from $31 \%$ to $61 \%$ survival to discharge. ${ }^{2,9,12,13}$ Differences in survival likely reflect differences between institutions in patient selection, indication for ECMO, timing of initiation of ECMO support, ECMO management, and availability of a rapid-response ECMO team. Center-specific case volume may impact survival as well, although there are no data in the literature to support this hypothesis. Higher survivals in our series may reflect the high proportion of patients cannulated for ECMO with an easily reversible cause for decompensation, such as shunt thrombosis or tamponade. Comparison of our analysis and previously published series is difficult, because survival according to ECMO indication is not always reported. How- 
ever, in recent reports including patients with shunted single ventricle physiology supported with ECMO, the proportion of patients supported with ECMO for failure to wean from CPB (37\%-57\%) ${ }^{12,13}$ was substantially higher than in our series $(11 \%)$. The variation in the percentage of patients supported with ECMO for failure to wean from CPB may represent the use of different criteria for patient selection for ECMO support, which may substantially influence outcomes.

Hypoxemia as the ECMO indication was the strongest predictor of survival of all variables investigated. Among patients supported for hypoxemia, those whose events were precipitated by shunt-related problems (thrombosis or stenosis) had the best outcomes, with $83 \%$ surviving to discharge. The more favorable outcome for this group of patients compared with those supported with ECMO for cardiovascular collapse is likely related to the acute and reversible nature of the events precipitating clinical deterioration. This subset of patients typically has a sudden and precipitous clinical deterioration that can progress rapidly to cardiac arrest. Our ability to rapidly cannulate for ECMO in an urgent situation, including during CPR, ${ }^{1}$ allowed us to stabilize these patients for diagnostic catheterization and/or shunt thrombectomy. Once the shunt obstruction was relieved, many of these patients could be easily weaned from ECMO because most patients in this group had preserved ventricular function.

Survival to discharge was significantly lower for patients supported with ECMO for hypotension or sudden cardiac arrest. This group represents a more diverse set of patients, few of whom had a discreet, easily reversible cause for their arrest or deterioration, such as was the case for patients with shunt thrombosis. Low cardiac output syndrome, cardiac arrest, and inability to wean from CPB are the most frequently cited indications for ECMO in patients with a shunted single ventricle in the literature. Although a subset of these patients may have CPB-related myocardial dysfunction that will improve with myocardial rest, others have either irrecoverable myocardial dysfunction or physiology that will not allow weaning from mechanical support despite an adequate technical repair. For example, 7 of 8 patients with PA or a variant did not survive to discharge in our series. Three of these patients had PA/IVS with RVDCC (1 patient with aortocoronary atresia), and significant myocardial ischemia developed after BT shunt placement. These patients with a baseline level of coronary ischemia may have further myocardial injury on ECMO because drainage of the right atrium via the ECMO cannula may decompress the right ventricle, thereby reducing coronary perfusion pressure, possibly precluding weaning from ECMO. Patients with PA/IVS and RVDCC who are managed with staged palliation have the highest risk of mortality after BT shunt placement. ${ }^{14}$ Three patients with PA variants who underwent BT shunt and pulmonary outflow tract patch had significant pulmonary regurgitation resulting in a retrograde circular shunt and difficulty weaning from ECMO. These patients, despite adequate technical repairs, had physiology that would not allow weaning from ECMO.

Of 13 patients catheterized in this study, only 4 had operative interventions based on findings at catheterization that subsequently allowed weaning from ECMO support. Of these 4 patients, 3 survived to discharge, all of whom had been cannulated for hypoxemia. The 1 patient cannulated for circulatory collapse who underwent operative intervention based on catheterization data was weaned from ECMO but did not survive to discharge. This also suggests that technically inadequate surgical results did not lead to ECMO in patients supported for indication of circulatory collapse.

Previous reports of patients with a shunted single ventricle who were supported with ECMO have suggested that mortality is higher among patients who have their shunt occluded on ECMO. ${ }^{9}$ Occlusion of all flow into the pulmonary arteries of neonatal piglets undergoing $\mathrm{CPB}$ is associated with increased pulmonary vascular resistance, increased alveolar-arterial $\mathrm{O}_{2}$ gradients, and decreased pulmonary compliance. ${ }^{15}$ Other authors have proposed that the increased mortality among patients who have complete shunt occlusion is related to pulmonary injury from ischemia-reperfusion. ${ }^{16} \mathrm{We}$ have therefore avoided complete occlusion of the BT shunt. However, in the subset of patients who continue to have inadequate systemic perfusion, as demonstrated by delayed lactate clearance despite increasing ECMO flows, we will narrow the shunt using 1 or more surgical clips. In this series, significantly more nonsurvivors than survivors had their shunts clipped. However, we hypothesize that the need for shunt clipping is a marker of severity of illness and is associated with increased mortality for that reason. Shunt clipping was required more frequently in patients cannulated for cardiovascular collapse.

\section{Limitations}

This study is limited primarily by its retrospective nature. In addition, generalizability of outcomes is limited by differences between institutions in the timing of ECMO initiation, attempts to wean from $\mathrm{CPB}$, and ECMO management.

\section{Conclusions}

Survival to discharge in this series of 44 patients with shunted single-ventricle physiology who were supported with ECMO was $48 \%$, comparable to the overall survival for cardiac ECMO in our institution. Thus, we do not consider shunted single-ventricle physiology to be a contraindication to ECMO support. The major determinant of outcome in this series is the indication for ECMO, with significantly greater survival to discharge for patients cannulated for hypoxemia. This likely reflects the readily reversible nature of the cause for deterioration in the majority 
of these patients. Mortality was higher among patients who had their shunts partially occluded, although this correlates with the indication for cannulation, suggesting that shunt occlusion is a marker for severity of illness.

\section{References}

1. Duncan BW, Ibrahim AE, Hraska V, del Nido PJ, Laussen PC, Wessel $\mathrm{DL}$, et al. Use of rapid-deployment extracorporeal membrane oxygenation for the resuscitation of pediatric patients with heart disease after cardiac arrest. J Thorac Cardiovasc Surg. 1998;116:305-11.

2. Kolovos NS, Bratton SL, Moler FW, Bove EL, Ohye RG, Bartlett RH, et al. Outcome of pediatric patients treated with extracorporeal life support after cardiac surgery. Ann Thorac Surg. 2003;76:1435-41; discussion 1441-2.

3. Morris MC, Ittenbach RF, Godinez RI, Portnoy JD, Tabbutt S, Hanna $\mathrm{BD}$, et al. Risk factors for mortality in 137 pediatric cardiac intensive care unit patients managed with extracorporeal membrane oxygenation. Crit Care Med. 2004;32:1061-9.

4. Alsoufi B, Shen I, Karamlou T, Giacomuzzi C, Burch G, Silberbach $\mathrm{M}$, et al. Extracorporeal life support in neonates, infants, and children after repair of congenital heart disease: modern era results in a single institution. Ann Thorac Surg. 2005;80:15-21; discussion 21.

5. Extracorporeal Life Support Registry Report. Ann Arbor, MI: Extracorporeal Life Support Organization; 2004.

6. Montgomery VL, Strotman JM, Ross MP. Impact of multiple organ system dysfunction and nosocomial infections on survival of children treated with extracorporeal membrane oxygenation after heart surgery. Crit Care Med. 2000;28:526-31.

7. Kulik TJ, Moler FW, Palmisano JM, Custer JR, Mosca RS, Bove EL, et al. Outcome-associated factors in pediatric patients treated with extracorporeal membrane oxygenator after cardiac surgery. Circulation. 1996;94:II63-8.
8. Walters HL 3rd, Hakimi M, Rice MD, Lyons JM, Whittlesey GC, Klein MD. Pediatric cardiac surgical ECMO: multivariate analysis of risk factors for hospital death. Ann Thorac Surg. 1995;60:329-36; discussion 336-7.

9. Aharon AS, Drinkwater DC Jr, Churchwell KB, Quisling SV, Reddy VS, Taylor M, et al. Extracorporeal membrane oxygenation in children after repair of congenital cardiac lesions. Ann Thorac Surg. 2001;72: 2095-101; discussion 2101-2.

10. Ungerleider RM, Shen I, Yeh T, Schultz J, Butler R, Silberbach M, et al. Routine mechanical ventricular assist following the Norwood procedure-improved neurologic outcome and excellent hospital survival. Ann Thorac Surg. 2004;77:18-22.

11. Hintz SR, Benitz WE, Colby CE, Sheehan AM, Rycus P, Van Meurs KP. Utilization and outcomes of neonatal cardiac extracorporeal life support: 1996-2000. Pediatr Crit Care Med. 2005;6:33-8.

12. Ravishankar C, Dominguez TE, Kreutzer J, Wernovsky G, Marino BS, Godinez R, et al. Extracorporeal membrane oxygenation after stage I reconstruction for hypoplastic left heart syndrome. Pediatr Crit Care Med. 2006;7:319-23.

13. Pizarro C, Davis DA, Healy RM, Kerins PJ, Norwood WI. Is there a role for extracorporeal life support after stage I Norwood? Eur J Cardiothorac Surg. 2001;19:294-301.

14. Guleserian KJ, Armsby LB, Thiagarajan RR, del Nido PJ, Mayer JE Jr. Natural history of pulmonary atresia with intact ventricular septum and right-ventricle-dependent coronary circulation managed by the singleventricle approach. Ann Thorac Surg. 2006;81:2250-7; discussion 2258.

15. Chai PJ, Williamson JA, Lodge AJ, Daggett CW, Scarborough JE, Meliones JN, et al. Effects of ischemia on pulmonary dysfunction after cardiopulmonary bypass. Ann Thorac Surg. 1999;67:731-5.

16. Jaggers JJ, Forbess JM, Shah AS, Meliones JN, Kirshbom PM, Miller $\mathrm{CE}$, et al. Extracorporeal membrane oxygenation for infant postcardiotomy support: significance of shunt management. Ann Thorac Surg. 2000;69:1476-83. 